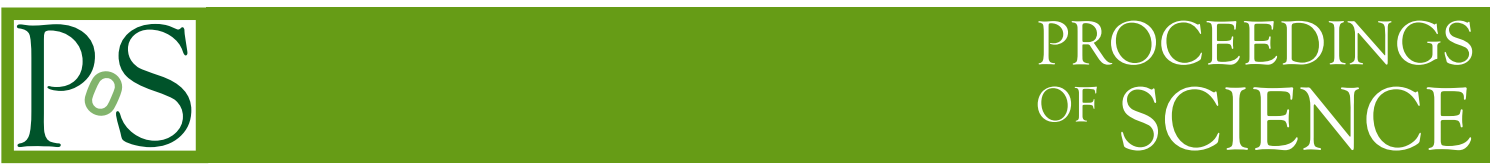

\title{
Performance of the ATLAS Tau Trigger in Run 2
}

\author{
Geert-Jan Besjes* \\ On behalf of the ATLAS collaboration \\ Niels Bohr Institute, University of Copenhagen \\ Blegdamsvej 17, DK-2100 Copenhagen, Denmark \\ E-mail: geert-jan.besjes@cern.ch
}

Tau leptons are used in a range of important ATLAS physics analyses, including searches for SM Higgs boson decays to fermions and measurements of the Higgs-fermion couplings, searches for Higgs boson partners, and heavy resonances that decay into pairs of tau leptons. Events for analyses are provided by a number of single and di-tau triggers, as well as triggers requiring tau leptons in combination with other objects. As the luminosity of proton-proton collisions at the LHC is going to exceed the design value of $10^{34} \mathrm{~cm}^{-2} \mathrm{~s}^{-1}$ in Run 2, the tau trigger strategies have become more sophisticated than in Run 1. Topological selections at the first trigger level, fast tracking algorithms and improved identification requirements are the main developments to allow a large program of physics analyses with tau leptons. The performance of the ATLAS tau trigger during the 2015 and early 2016 data taking is discussed, as well as plans for further developments envisaged during Run 2.

38th International Conference on High Energy Physics

3-10 August 2016

Chicago, USA

${ }^{*}$ Speaker. 


\section{Introduction}

In many Standard Model and beyond-the-Standard-Model processes, including the decay of the Higgs boson, tau leptons are produced and form a key experimental signature. Tau leptons decay either hadronically (65\%) or into other leptons. Due to their short lifetimes, they decay before the detector is reached. Tau triggers aim to detect hadronic tau decays; the leptonic modes are covered by electron and muon triggers. These hadronic decays must be distinguished from quark- and gluon-initiated jets, a challenge due to their similar detector signatures. Tau triggers exploit the fact that a typical tau decay includes one or three tracks, coming from charged pions, in a collimated jet that originated from a displaced vertex.

The Run-2 ATLAS trigger system is designed to reduce the event rate from the collision rate of $40 \mathrm{MHz}$ to approximately $1 \mathrm{kHz}$ that is written to storage, and includes dedicated bandwith for hadronic tau triggers. The increase in centre-of-mass energy and instantaneous luminosity poses a significant challenge given the time and bandwidth constraints of the system. As a result, the tau trigger strategy has changed with respect to Run 1.

\section{The ATLAS Trigger System}

The ATLAS trigger used in Run 2 is a two-level system [1]. Level 1 (L1), the first level, consists of hardware that identifies regions of interest (RoIs) using information from the calorimeters and the muon spectrometer. The High Level Trigger (HLT) receives these RoIs at a rate of up to 100 $\mathrm{kHz}$. It then performs object reconstruction using algorithms that are as close as possible to their counterparts used in offline reconstruction.

Compared to Run 1, the trigger system has undergone several significant changes to deal with the increased LHC energy and luminosity [2]. First, the bandwidth at both L1 and the HLT has increased. Second, a new central trigger processor (CTP) at L1 allows for a greater number of trigger thresholds. Furthermore, a new topological trigger module allows for more complicated selections on multiple RoIs, e.g. using their angular separation. Finally, the former Level-2 trigger and Event Filter have been merged into a single HLT.

\section{Tau trigger strategy}

The L1 tau triggers in ATLAS identify RoIs by applying selections on the sum of the energy recorded by groups of $2 \times 2$ calorimeter towers, using information from both the electromagnetic and hadronic calorimeters. Optionally, a selection on the energy deposits in the annulus around this core is used to reject signatures from QCD jets. Topological selections, using e.g. angular separation or overlap removal, between L1 tau objects and other objects, such as electrons, missing transverse energy or other taus can also be employed.

All identified RoIs are passed on to the HLT, where their initial energy and position measurements are first refined. A selection on the deposited energy is then applied on an estimate obtained from pile-up robust topo-clustering algorithm [3]. Taus are further distinguished from jets through the use of a fast tracking algorithm and selections on the identified track multiplicities. One or three tracks in a core region are required, and a maximum of 1 track is allowed in the annulus around this core. Only tracks with a transverse momentum greater than $1 \mathrm{GeV}$ are considered. 
Finally, full track reconstruction is performed. A Boosted Decision Tree (BDT) that relies on tracking and calorimeter shower shape information is then used to identify the final candidates. The BDT is tuned using the same information as its offline counterpart [5]. Three working points are defined to select samples of varying purity.

\section{Performance in Run 2}

An important test for the tau triggers is to check their robustness with increasing numbers of simultaneous soft collisions, so-called pile-up. Fig. 1 shows the L1 rates of several triggers as a function of the instantaneous luminosity; the rates' linearity reflects the pileup robustness of the trigger. The L1 objects are indicated as 'TAU', 'EM' (electron), ' $\mathrm{J}$ ' (jet) and 'XE' (missing transverse energy). The digits before these names indicate a multiplicity, those after them an energy requirement in GeV. Additionally, 'IM' refers to an isolation requirement and 'HI' to a veto on the presence of hadronic energy and an isolation selection. The trigger

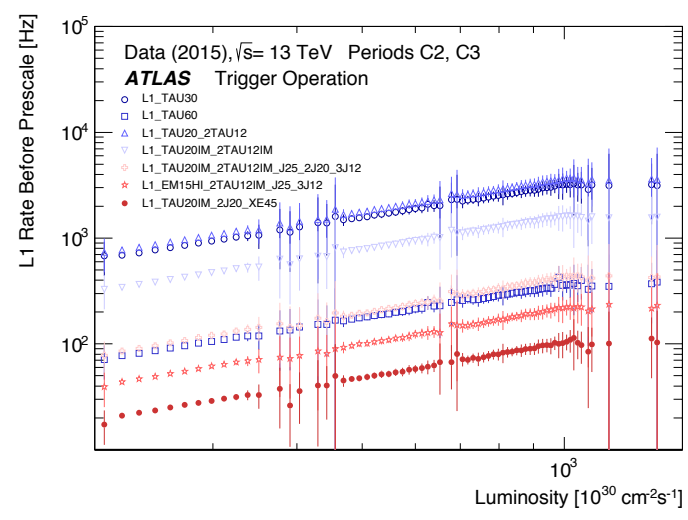

Figure 1: Level 1 trigger rates for various single and combined L1 tau trigger items [4]. Further details are given in the text.

L1_TAU20_2TAU12IM thus selects one tau RoI with $20 \mathrm{GeV}$ and one tau RoI with $12 \mathrm{GeV}$, both isolated. The data were collected at a centre-of-mass energy of $13 \mathrm{TeV}$ between 6 and 12 July 2015, using a bunch spacing of $50 \mathrm{~ns}$.

Fig. 2 shows the BDT score distributions of online tau candidates passing the HLT trigger 'tau25_medium' in both data and Monte Carlo simulation. The medium working point was chosen to have an efficiency of $\sim 95 \%$ w.r.t. selected offline tau candidates. The measurements have been obtained using a tag-and-probe method in $Z \rightarrow \tau\left(\rightarrow v_{\tau} v_{\mu} \mu\right) \tau\left(\rightarrow \tau_{\text {had }} v_{\tau}\right)$ events, where one tau lepton decays via a muon that is tagged by the muon trigger. The second probe tau is then fully reconstructed and the tau trigger's decision verified. The underlying distributions of the variables in this BDT also show excellent agreement [4]. The background candidates are obtained from two samples, enriched in $W \rightarrow \mu v_{\mu}+$ jets and in multijet events, where candidate tau leptons are likely to be misidentified quark- or gluon-initiated jets.

From such events the trigger efficiency with respect to the offline selection can be measured as a function of $p_{\mathrm{T}}$, shown in fig. 2. Trigger efficiencies can then be compared between data and simulation, and corrections (scale factors) derived. The methodology used to obtain the the efficiencies is further described in [5].

\section{Prospects}

The LHC is foreseen to collide proton beams at even higher instantaneous luminosities in the future. As a result, the tau trigger bandwidth will more likely saturate. One of the possible significant improvements that will lead to a rate reduction, at minimal cost to signal efficiency, is to reject 

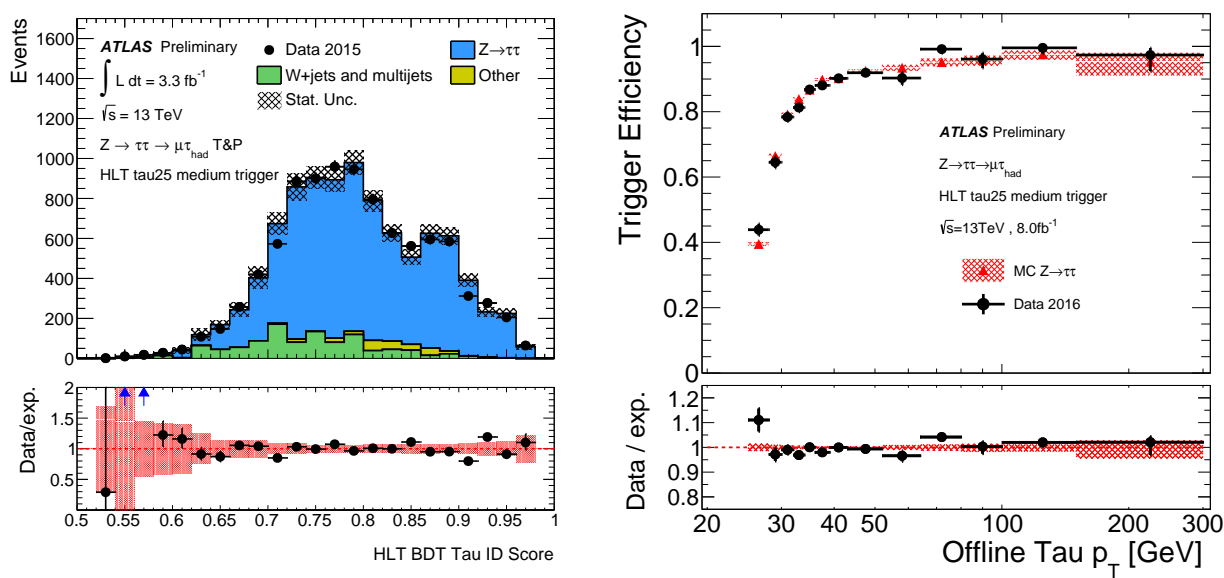

Figure 2: The online HLT BDT score (left) and the trigger efficiency as a function of the tau transverse momentum (right) for the tau25_medium trigger [4].

events likely to contain quark- or gluon-initiated misidentified candidates by applying analysis event selection requirements at trigger level that target multi-jet topologies. The commissioning of such topological triggers is foreseen in late 2016.

The ATLAS experiment is currently in the process of being upgraded with a Fast Tracker (FTK) custom hardware system that will provide tracks directly after Level 1 decisions through the use of trained pattern recognition banks. The FTK will allow the HLT algorithms that identify tau lepton candidates to directly use these tracks, rather than having to perform a fitting procedure in the RoIs first. This tracking information can be exploited before calorimeter energy deposits are used, reducing the impact of the energy resolution on the trigger efficiency. The first triggers are planned to be operational in 2017 and are foreseen to improve triggering on processes such as $H \rightarrow \tau \tau$ [6].

\section{Conclusion}

The ATLAS tau triggers have undergone significant changes in preparation for Run 2, including a redesigned HLT selection and an improved BDT algorithm. Analysis of data collected by the ATLAS experiment has shown that the trigger is performing efficiently. The online distributions of kinematic variables and the HLT BDT score distributions extracted from $Z \rightarrow \tau \tau$ events in data are well-modeled by simulation. Further studies of the efficiency of the tau trigger will continue as more data is collected and trigger scale factors will be estimated. In the longer term, the use of topological tau triggers and of FTK-based triggers is foreseen to reduce the rate of the tau triggers without significantly decreasing signal efficiency.

\section{References}

[1] ATLAS Collaboration, JINST 3 (2008) S08003. ATLAS Collaboration, CERN-LHCC-2013-018 (2013).

[2] ATLAS Collaboration, ATLDAQ-PROC-2015-018 (2015).

[3] ATLAS Collaboration, Eur. Phys. J. C 75 (2015) 303.

[4] ATLAS Collaboration, https://twiki.cern.ch/twiki/bin/view/AtlasPublic/TauTriggerPublicResults.

[5] ATLAS Collaboration, ATL-PHYS-PUB-2015-025 (2015).

[6] ATLAS Collaboration, ATLAS-TDR-021 (2013). 